\title{
Application of Multi Slice Spiral CT Combined with Head and Neck Digital Subtraction Angiography in the Diagnosis of Head and Neck Vascular Diseases
}

\author{
Taoping Zhou \\ Department of Radiology, Qujing Second People's Hospital, Qujing, Yunnan 655000, China \\ 1045013977@qq.com
}

Keywords: Head and neck; Arterial disease; 128 slice spiral CT; Digital subtraction angiography

\begin{abstract}
To evaluate the value of 128 slice spiral CT combined digital subtraction angiography in the diagnosis of head and neck vascular diseases. Materials and methods: from March 2016 to December 2016 during the screening in the Institute of clinical diagnosis of subarachnoid hemorrhage (21 cases), cerebral hemorrhage (15 cases), cerebral infarction (49 cases), transient ischemic attack (15 cases), frontal mass ( 1 cases), neck mass ( 2 cases) and accept the 128 slice CT digital subtraction angiography (DSCTA) in 103 cases of patients, the imaging data were retrospectively analyzed and compared with that of DSA. The head neck vascular diagnosis experienced 2 attending doctors to assess vascular imaging quality, vascular stenosis and plaque nature, aneurysm, arteriovenous malformation, vascular variation. Results: the neck vascular one or a plurality of different soft and hard plaque caused by different degree of luminal stenosis and occlusion (51 cases), abnormal blood vessels (16 cases), unilateral vertebral artery dysplasia (3 cases), aneurysm (22 cases), arteriovenous malformation (3 cases), carotid body tumors ( 2 cases), normal (6 cases). Conclusion 128 slice digital subtraction angiography (DSCTA) can effectively remove bone parts of vascular lesions, accurate clear images of size, shape and range and its relationship with the surrounding tissue for clinicians, and is non-invasive, rapid, simple, high safety and for head and neck vascular stenosis and soft plaque vascular variation, dysplasia, aneurysm, application of venous malformation diagnosis for accuracy, high reliability and clinical treatment is higher, it is worthy of popularization and application.
\end{abstract}

\section{Introduction}

Head and neck vascular lesions in cerebral ischemic or hemorrhagic diseases are the main causes, if you do not take effective measures in a timely manner will lead to further treatment of vascular stenosis or occlusion and rupture, with high morbidity and mortality, causing a huge threat to human health, to improve the accuracy to improve early treatment and prognosis of patients the clinical diagnosis[1][2]. Because of the conventional digital subtraction angiography (DSA) has high resolution, and can be used as a clinical treatment for head and neck vascular diseases, has long been regarded as the diagnosis and treatment of head and neck vascular disease of the "gold standard", but it is invasive, high cost, high radiation, poor reproducibility, and only display the spatial structure of vascular cavity of patients, can not accurately assess the vascular wall and surrounding lesions 3. In recent years, with 64 rows of 128 slice spiral CT digital subtraction angiography (DSCTA) in clinical application, it can effectively remove bone, simple operation, by volume rendering (VR), maximum intensity projection (MIP), multi planar reconstruction (MPR), surface recombination (CPR) after treatment, can be obtained clear image information of patients and lesions around the vascular wall, plays an important role to develop head and neck vascular disease diagnosis and treatment, in the diagnosis of head and neck vascular diseases have gradually replaced the conventional DSA examination trend. The study of our hospital 128 shice spiral CT scanning digital subtraction angiography. The clinical data were analyzed retrospectively, to explore its application value in the head and neck vascular diseases, the relevant data and results are reported as follows. 
General Information. The object of this study is: a retrospective analysis of March 2016 December 2016 in the Institute of clinical diagnosis of subarachnoid hemorrhage (21 cases), cerebral hemorrhage (15 cases), cerebral infarction (49 cases), transient ischemic attack (15 cases), frontal mass ( 1 cases), cervical mass ( 2 cases) and underwent 128 slice spiral CT digital subtraction angiography (DSCTA) examination of the patients (103 cases). There were 68 men and 35 cases of female patients, age (1880 years old, mean age $(49+8.5$ years old).

Inspection Method. The United States GE company production of 128 small gem CT (OptimaCT660), using double tube high pressure syringe through right cubital vein injection of nonionic contrast agent (iohexol, $350 \mathrm{mg} / \mathrm{mL}$ ) $60 \mathrm{~mL}$, additional injection of saline 40mL. Before the scan, the clinical symptoms will check the process and inspection process may appear in detail to inform patients for patients closely cooperate with the inspection work, the special head head in patients with small scanning and bandage, with eyes closed and keep quiet breathing, strict brake. Advanced plain (get thin plain diagnosis needs from the aortic arch vertex scan image), after the pre scanning technology to complete the monitoring scan (trigger CT value is $100 \mathrm{HU}$, the delay time for $4 \sim 5 \mathrm{~s}$, as the starting point with the scanning position monitoring plane scan range from the descending aorta, aortic arch: top of the head), $10 \mathrm{~s}$ after injection of contrast with the monitor scan, reaches the preset threshold value after the corresponding time delay for enhanced scan, and constant, plain and enhanced the position, level and ensure the consistent view of the center. Scanning parameters: voltage $120 \mathrm{kV}$, current of $320 \mathrm{~mA}$, pitch 0.984:1; detector collimation width of $0.625 \mathrm{~mm}$, matrix $* * 512 * 512$; . The analysis of the original data to GE ADW4.6 workstation after reconstruction techniques including volume rendering (VR), maximum intensity projection (MIP) and multi level recombinant (MPR) and surface recombination (CPR) and CT-DSA mature application (DSA) technology, through the special operation of cervical vertebra and skull silhouette, will all cut off, display only the carotid artery and vertebral artery, Willis ring, the head and neck Department DSA imaging.

Evaluating Indicator. According to the image quality in three levels: goodwellbad of bilateral carotid artery and bilateral vertebral basilar artery, bilateral anterior cerebral artery, middle cerebral artery, bilateral middle cerebral arteries after subtraction quality evaluation system (clear image without artifact subtraction vascular wall smooth, can well meet the diagnostic requirements (good); clear image without artifact subtraction, the existence of small vascular wall defect, basically can make a diagnosis), poor (image artifacts, subtraction vascular defects, unable to diagnose) three level assessment

(2) To assess the degree of stenosis: according to the North American symptomatic carotid endarterectomy (NASCET) standard test[4], carotid stenosis rate $=1$ : residual stenosis of distal stenosis diameter / diameter $\mathrm{x} 100 \%$, intracranial artery stenosis rate $=1$ residual stenosis diameter stenosis / proximal diameter * $100 \%$; the degree of carotid artery stenosis divided into 4 levels: mild stenosis $(0 \% \sim 29 \%)$; moderate stenosis $(30 \% \sim 69 \%)$; severe stenosis $(70 \% \sim 99 \%)$; block $(100 \%)$.

(3)To assess the nature of plaque[5]: lipid plaque: < 60HU; fibrous plaque: from 60 to $130 \mathrm{HU}$; calcified plaque: $>130 \mathrm{HU}$.

Evaluation Method . Head neck vascular diagnosis experienced 2 attending doctors to evaluate.

\section{Result}

After treatment, the image quality was excellent in 93 cases $(90.3 \%)$, good in 8 cases $(7.8 \%)$, poor in 2 cases (1.9\%), and the excellent and good rate was $98.1 \%$.

Patients with CTA examination, 6 cases were normal, 97 cases of abnormal blood vessels in the head and neck, one or a plurality of different soft and hard plaque caused by different degree of luminal stenosis and occlusion (51 cases), abnormal blood vessels (16 cases), unilateral vertebral artery dysplasia ( 3 cases), aneurysm ( 22 cases), arteriovenous malformation ( 3 cases), carotid body tumor $(2$ cases). The degree of arterial stenosis in this group of patients were detected arterial 
stenosis in 42, of which 12 artery mild stenosis (28.6\%), moderate stenosis 16 (38.1\%), severe stenosis in 9 segments $(21.4 \%), 5(11.9 \%)$ total occlusion.

\section{Discuss}

Ischemic Stroke. Ischemic stroke is a common cerebrovascular disease, which accounts for $60 \%$ to $80 \%$ of all cerebrovascular diseases[6]. This study shows that 128 slice spiral CT digital subtraction angiography has high success rate, noninvasive, rapid, simple, high security, and through VR, MIP, MPR and CPR DSCTA postprocessing technology can clearly show the head and neck stenosis plaque location, scope, nature and the degree of stenosis and combined with the original axial images comprehensive analysis of diagnostic accuracy, can provide important reference information for clinical diagnosis and treatment of patients, and a large number of domestic reported DSCTA results can accurately reflect the degree of vascular stenosis[7]. 91.5\% sensitivity of 64 slice CTA in diagnosis of vascular stenosis and occlusion, the specificity was 97.5\% . 2.5\% false positive, $8.5 \%$ false negative. At present, the advantages of DSCTA in the diagnosis of head and neck vascular stenosis have been recognized by medical science [1][2][3][6][7]. The inadequacy of this study: 1. To compare with the results of DSA, conventional digital subtraction angiography (DSA) has high resolution, and can be used as a clinical treatment for head and neck vascular diseases, has long been regarded as the diagnosis and treatment of head and neck vascular disease of the "gold standard", but it is invasive, high cost, high radiation, poor reproducibility, and can only display the spatial structure of the vascular cavity of patients, can not accurately assess the vascular wall and surrounding lesions. In addition, there are reports that, the postoperative complications of DSA in the range from $1.5 \%$ to $2 \%$ [9], the follow-up treatment of patients will have some interference. CT perfusion imaging (CTP) at the same time, CTP can be used to analyze the blood perfusion of brain tissue, combined with CTA can also understand the situation of vascular stenosis, contribute to the diagnosis and treatment of cerebral infarction.

Aneurysm. Aneurysm is the primary cause of subarachnoid hemorrhage, occurred in the cerebral artery branch, cross and sharp corners, especially the Willis ring of the anterior communicating artery region, the group of 22 cases $(30.56 \%)$ occurred here, shear stress, and the variation of Willis circle caused by dynamic change of stroke and pressure, promote the occurrence of cerebral aneurysm. Patients with intracranial aneurysm once formed after the development of the size and shape remain unchanged, the formation of large aneurysm occlusion, thrombosis and bleeding, the bleeding probability is about $90 \%$ when the first bleeding ruptured, the fatality rate is about $15 \%$, such as second bleeding, mortality rate can be increased to about $45 \%$, the diagnosis of aneurysms to reduce the fatality rate is important[10][11]. The data analysis shows that 128 slice spiral CT digital subtraction angiography image to complete and clearly show the Willis ring and 4 $\sim 5$ grade cerebral vascular branches, and multi angle and multi direction. With the existence of internal aneurysm and the location of the aneurysm, aneurysm, aneurysm neck and calcification, provide a true the relationship between aneurysm and surrounding blood vessels and bone, the diagnosis reliability, accuracy and provide an effective basis for accurate anatomic localization for clinical treatment, has been widely used in clinical (neuro surgery, interventional radiology), also gradually gained the trust of clinicians. The preoperative CTA findings of the patients were consistent with the intraoperative findings and the DSA findings of embolization, and the coincidence rate was above $96 \%$. Consistent with the literature report [12], the sensitivity and specificity of the CTA in the diagnosis of intracranial aneurysms were very high, the sensitivity was $92 \%$ to $94 \%$, and the specificity was from $91 \%$ to $100 \%$. Zhang Longjiang, Lu Guangming and other research results show that[13]. The sensitivity and specificity of CTA diagnosis of aneurysm were $95 \%, 100 \%$; less than $3 \mathrm{~mm}$ in aneurysm diagnosis sensitivity and specificity were $80 \%$ and $100 \%$, there is a good correlation between detection of CTA aneurysm average long axis and short axis and the size of the aneurysm neck and 3D-DSA technology.

Arteriovenous Malformation (AVM). Arteriovenous malformation (AVM) is a congenital central nervous system vascular dysplasia, the group detected 3 cases of arteriovenous malformation, CTA image postprocessing technique can clearly display the AVM artery, nidus and 
draining veins, and can clearly show the spatial relationship between the adjacent structure and focus, a clear judgment AVM bleeding caused by arteriovenous malformation, improve the diagnostic coincidence rate [11]. He Xihua, Zheng Xiao Lin reports points to CTA diagnosis of arteriovenous malformation coincidence rate was $95.45 \%[14]$, the surgical and interventional physicians to understand the anatomy of the lesions and to develop treatment plans are of great help.

Vascular Variation CTA. CTA can clearly display the vascular variation of various congenital variation of head and neck arteries, such as head and neck artery origin, shape variation, side A1 segment of anterior cerebral artery absence or dysplasia by contralateral anterior cerebral artery blood supply: one side or both sides of P1 segment of posterior cerebral artery absence or dysplasia by ipsilateral posterior communicating branch of direct continuation of P2 segment.

In summary, 128 slice spiral DSCTA can effectively remove the influence of the skull base and cervical vertebrae of vascular imaging, image clarity, and is non-invasive, rapid, simple, high safety and soft plaque caused by neck vascular stenosis and vascular variation, dysplasia, aneurysm, advantages of high accuracy and reliability of diagnosis venous malformations and other diseases, and provide effective basis and accurate anatomic localization for clinical treatment, it is worthy of popularization and application.

\section{Reference}

[1] H.Q Lai,Y.Q:Modern Medical Imaging. Vol. 25(2016) No.3, p.472-474. (In Chinese)

[2] H.F Shi, P. Han and F.Y Wu: Journal of Clinical Radiology, Vol. 30(2011) No.9, p.1274-1277. (In Chinese)

[3] Y. Wei, P Chen and C. Tan: Journal of Practical Radiology, (2015) No.10, p. 1671-1674. (In Chinese)

[4] N Engl J Med:North American Symptomatic Carotid Endarterectomy Trial Collaborators, Vol. 325(1991) p. 445-453.

[5] Schroeder S,Kopp AF and Baumbach A : Eur RadioL, Vol. 11(2001), p. 1607-1611.

[6] H.L.Lu: Imaging and intervention, Vol. 21(2014) No.3, p.2095-2096. (In Chinese)

[7] X.J Du, X.X. Tang: Chinese Journal of Integrated Traditional Chinese and Western Medicine, Vol. 11(2014) No.12, p. 1349-1351. (In Chinese)

[8] J.J. Zhou, K.R. Zhou and Z.W Chen: Radiologic Practice, Vol. 21(2006) No.4, p. 336-339. (In Chinese)

[9] H.D. Min, W.H. Xie and S.Y. Yao: Chinese Journal of Medical Imaging, Vol. 215(2013) No.1, p173-176. (In Chinese)

[10]ZH. Xue,Zh.H. Sun and B.N. Xu:Journal of PLA Medical College, (2013) No.7, p. 715-718. (In Chinese)

[11]X.L Niu, M. Yu and Zh.M Wang: Journal of disease surveillance and control, Vol. 5(2011) No.3, p. 271-272. (In Chinese)

[12] Y.Yan, K.P. Chen:Journal of Qiqihar Medical College, Vol. 32(2011) No.7, p. 1075 -1076. (In Chinese)

[13]L.J. Zhang, X. Chai, G.M. Lu: Journal of clinical radiology, Vol. 28(2009) No.10, p. 1349-1352. (In Chinese)

[14]X.H. He, X.L. Zheng: Chinese Journal of Medical Sciences, Vol. 13(2015) No.5, p. 172-175. (In Chinese) 